\title{
Objective falls-risk prediction using wearable technologies amongst patients with and without neurogenic gait alterations: a narrative review of clinical feasibility
}

\author{
Callum M. W. Betteridge ${ }^{1,2,3,4}$, Pragadesh Natarajan ${ }^{1,2,3,4}$, R. Dineth Fonseka ${ }^{1,2,3,4}$, Daniel Ho ${ }^{1,2,3,4}$, \\ Ralph Mobbs ${ }^{1,2,3,4}$, Wen Jie Choy ${ }^{1,2,3,4}$
}

${ }^{1}$ Department of Medicine, University of New South Wales, Sydney, Australia; ${ }^{2}$ NeuroSpineClinic, Suite 7 Level 7, Prince of Wales Private Hospital, Randwick, Australia; ${ }^{3}$ NeuroSpine Surgery Research Group, Sydney, Australia; ${ }^{4}$ Wearables and Gait Assessment Group, Sydney, Australia Contributions: (I) Conception and design: CMW Betteridge, R Mobbs, P Natarajan; (II) Administrative support: CMW Betteridge, R Mobbs, WJ Choy, P Natarajan, RD Fonseka; (III) Provision of study materials or patients: CMW Betteridge, D Ho; (IV) Collection and assembly of data: CMW Betteridge, D Ho; (V) Data analysis and interpretation: CMW Betteridge, P Natarajan, RD Fonseka; (VI) Manuscript writing: All authors; (VII) Final approval of manuscript: Sydney, Australia All authors.

Correspondence to: Callum M. W. Betteridge. NeuroSpine Clinic Suite 7, Level 7 Prince of Wales Private Hospital Sydney, NSW, Australia; Department of Medicine, University of New South Wales, Sydney, Australia. Email: cbetteridgeresearch@gmail.com.

Objectives: The present narrative review aims to collate the literature regarding the current use of wearable gait measurement devices for falls-risk assessment in neurological and non-neurological populations. Thereby, this review seeks to determine the extent to which the aforementioned barriers inhibit clinical use.

Background: Falls contribute a significant disease burden in most western countries, resulting in increased morbidity and mortality with substantial therapeutic costs. The recent development of gait analysis sensor technologies has enabled quantitative measurement of several gait features related to falls risk. However, three main barriers to implementation exist: accurately measuring gait-features associated with falls, differentiating between fallers and non-fallers using these gait features, and the accuracy of falls predictive algorithms developed using these gait measurements.

Methods: Searches of Medline, PubMed, Embase and Scopus were screened to identify 46 articles relevant to the present study. Studies performing gait assessment using any wearable gait assessment device and analysing correlation with the occurrence of falls during a retrospective or prospective study period were included. Risk of Bias was assessed using the Centre for Evidence Based Medicine (CEBM) Criteria.

Conclusions: Falls prediction algorithms based entirely, or in-part, on gait data have shown comparable or greater success of predicting falls than existing stratification scoring systems such as the 10-meter walk test or timed-up-and-go. However, data is lacking regarding their accuracy in neurological patient populations. Inertial measurement units (IMU) have displayed competency in obtaining and interpreting gait metrics relevant to falls risk. They have the potential to enhance the accuracy and efficiency of falls risk assessment in inpatient and outpatient setting.

Keywords: Wearable technologies; inertial measurement units (IMU); gait; falls; neurological disease

Received: 16 February 2021; Accepted: 12 June 2021; Published: 20 October 2021.

doi: $10.21037 /$ mhealth-21-7

View this article at: https://dx.doi.org/10.21037/mhealth-21-7 


\section{Introduction}

Injuries are the fifth largest disease burden in Australia and falls alone account for $40 \%$ of injury-related admissions (1). A fall is an event in which a person inadvertently comes to rest on the ground (2). Falls are overrepresented in elderly populations and mostly occur during walking (3). A neurogenic gait alteration (NGA) is an alteration to gait due to abnormalities of the central or peripheral nervous system(s) resulting in changes to ambulation (4). The presence of an NGA doubles an elderly person's risk of falling by altering postural stability, limb dynamics, movement speed, symmetry and smoothness (5). Multiple sclerosis, Parkinson's disease, stroke, cerebral palsy, neurogenic claudication and peripheral neuropathy are common causes of NGA (3). Historically, tests like the Timed Up-and-Go (TUG), Berg-Balance-Scale (BBS), 10-Meter-Walk Test (10MWT) and Physiological Profile Assessment (PPA) were used for clinical assessment of fallsrisk. These 'test batteries' require the patient to perform compound movements that assess balance and coordination during walking and completing questionnaires on existing risk-factors. However, they have high inter- and intraobserver variability and only provide an overall falls-risk without identifying which gait metrics are contributing, or where to intervene $(6,7)$. With advances in gait-analysis technology, the gait perturbations prevalent in patients prone to falling can be assessed. Then, accurate capture of a patient's gait metrics in a clinical setting would allow the clinician to make unbiased recommendations for intervention based on the perturbations of gait displayed

Wearable devices capture the 'free-living' gait of unobserved and routine walking behaviours (8). Wearable sensor technology also enables for remote monitoring of many patient metrics by feeding data from small wearable devices to a database on a phone or cloud accessible by the patient and physician (9). For gait analysis, the most common device is a single Inertial Measurement Unit (IMU) or 'single-point' wearable at the pelvis or sternum near the centre of mass (COM), at the ankle or feet for more precise step-recognition, or at multiple points with integrated signals (multi-point wearable sensors) (10). IMUs comprise of at least an accelerometer and gyroscope, sometimes accompanied by magnetometer. Recent IMUs employ tri-axial accelerometers which measure in 3 axes, anteroposterior (AP), medio-lateral (ML) and vertical (VT) (11). IMUs can calculate some gait metrics such as velocity, distance, step and turning occurrences and durations (12), from raw inertial measurements (and accompanying noise). To become the gold-standard clinical falls-risk assessment tool, IMUs must accurately capture and process relevant gait metrics to create falls-risk profiles from short walking bouts (WBs) to generate falls-risk profiles. These falls-risk predictions derived from IMU-derived gait metrics are consequently compared to patient-report of falls over a set retrospective period of time, acquired through a questionnaire and/or interview. This requires prior understanding of literature using quantitative gait assessment comparing fallers and non-fallers to identify which gait parameters are implicated in falls (13). Recent reviews have found that, compared to non-fallers, fallers have reduced gait velocity $(\mathrm{GV})(13-17)$, cadence and step length (SL) $(13,16)$, increased dynamic sway (13) and more variability in gait speed (GSV), step time (STV) and step length (SLV) (16). Table 1 summarises the gait variables often used to compare fallers and age- and disease-matched non-fallers using these variables and four others, step width (SW), step count (SC), static sway and turning kinematics, that have not been reviewed. For explanation of gait metrics, see Figures 1-4. We present the following article in accordance with the Narrative Review reporting checklist (available at https://dx.doi.org/10.21037/mhealth-21-7).

\section{Rationale}

This review aims to investigate the application of wearable technology in capturing and processing relevant gait data for falls-risk analysis. In particular, this study aims to explore the presence of three barriers to the implementation of IMU devices in clinical falls-risk assessment: (I) accurately measuring gait metrics which are proven to be associated with high falls risk (mentioned above), (II) identifying differences in these metrics between groups of patients who have fallen, and those who have not, (III) accuracy of fallsprediction algorithms developed from IMU-collected gait metrics (Appendix 1).

\section{Objectives}

To perform a narrative review involving collection and critical appraisal of literature pertaining to the use of wearable devices in the prediction of falls, and identify factors limiting clinical implementation of IMU devices in falls-risk assessment. 
Table 1 Definitions of all gait metrics which were used from included studies

\begin{tabular}{|c|c|c|c|}
\hline Simple metric & Definition & Units & Derivative metrics \\
\hline GV & $\begin{array}{l}\text { Average distance travelled in the ambulant } \\
\text { direction per second. }\end{array}$ & $\begin{array}{l}\text { Meters per } \\
\text { second }\end{array}$ & GSV, the standard deviation of GV \\
\hline ST & $\begin{array}{l}\text { Average amount of time between two consecutive } \\
\text { contacts of the same foot with the ground }\end{array}$ & Seconds & STV, the standard deviation of ST \\
\hline SL & $\begin{array}{l}\text { Average distance between two consecutive } \\
\text { contacts of the same foot with the ground }\end{array}$ & Meters & SLV, the standard deviation of SL \\
\hline $\begin{array}{l}\text { Dynamic } \\
\text { balance }\end{array}$ & $\begin{array}{l}\text { Aberrant movement of the centre of mass within a } \\
\text { single gait cycle. Several ways to measure. }\end{array}$ & Several & $\mathrm{N} / \mathrm{A}$ \\
\hline $\begin{array}{l}\text { Static } \\
\text { balance }\end{array}$ & $\begin{array}{l}\text { Movement of the centre of mass whilst standing } \\
\text { still with feet together }\end{array}$ & Meters & $\mathrm{N} / \mathrm{A}$ \\
\hline $\begin{array}{l}\text { Turning } \\
\text { kinematics }\end{array}$ & $\begin{array}{l}\text { Number of steps taken, or time taken to complete } \\
\text { a } 180 \text {-degree turn }\end{array}$ & s & $\mathrm{N} / \mathrm{A}$ \\
\hline
\end{tabular}

See available online: https://cdn.amegroups.cn/static/public/mhealth-21-7-1.xlsx for more details. m, meters; s, seconds; m/s, meters per second; Hz, Hertz (number of steps per second); GV, gait velocity; ST, stride time; SL, stride length; GSV, gait speed variability; STV, stride time variability; SLV, stride length variability.

\section{Methods}

Review protocol registered on PROSPERO (Appendix 2).

\section{Eligibility}

Included studies comprised of:

(I) Cohort studies or case-control studies;

(II) Studies with 6 or greater month follow up;

(III) Studies conducting gait analysis with wearable technologies measuring relevant gait metrics and/ or used relevant gait metrics to assess falls-risk with analysis of diagnostic test accuracy;

(IV) Studies written in English and available in full-text.

It should be noted that information could not be presented from papers in which methodology was not adequately discussed, in particular if they failed to provide detailed description of data capture, processing, and statistical analysis. This resulted in one paper being discounted from qualitative synthesis.

\section{Information sources}

Databases used to search for articles were Medline, PubMed, Scopus and Embase. All articles with results discussed came from a structured literature search. A similar search strategy was used across all databases for consistency, done in February 2020.

PubMed example: (((acceleromet* OR gyrometer OR magnetometer OR gait analysis) AND (sensor OR tracker OR device OR detector OR wearable) AND (gait OR posture OR ambulation)) AND (falls risk OR fall risk OR fall prediction OR falls prediction)).

\section{Study selection}

Studies were collated in EndNote X9 and duplicates removed. Studies were then screened based on inclusion/ exclusion criteria by 2 independent reviewers (CB and $\mathrm{DH}$ ) on an online reviewing software by Rayyan (Qatar Computing Research Institute). Discrepancies were 


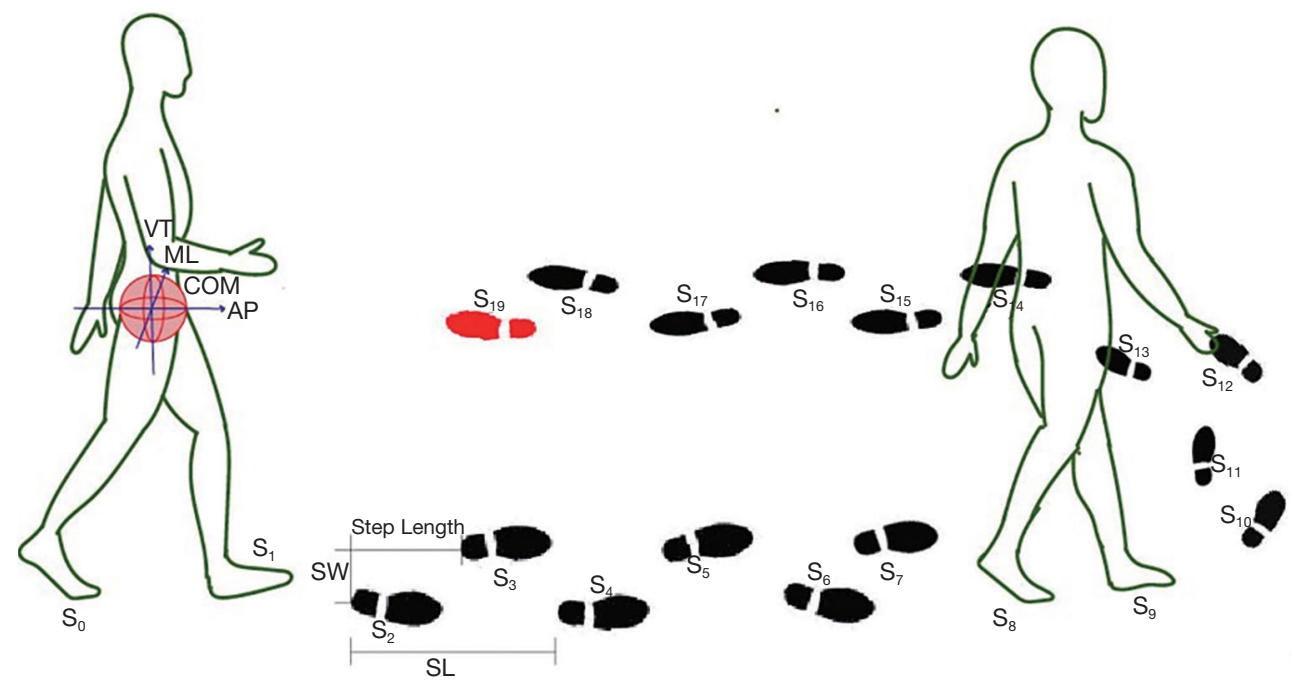

Figure 1 Gait bout. $S_{0}=$ step that begins measurement window, turning occurs from $S_{8}-S_{14}\left(7\right.$ steps). $S_{18}$ and $S_{19}$ represent a variation in step length. SL, stride length; SW, step width; VT, vertical; ML, medio-lateral; COM, Centre of motion; AP, antero-posterior; S (n), nth step.

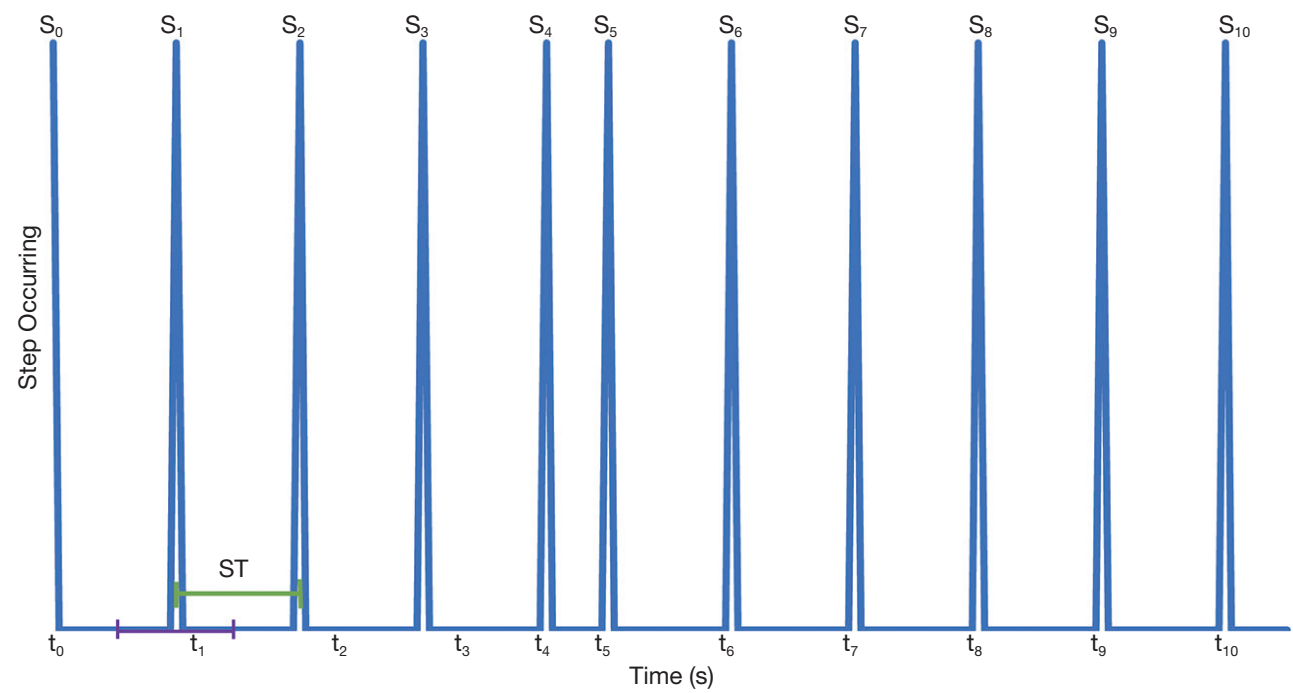

Figure 2 Timing of gait bout in Figure 1. S $(n)=n$th step, $t(n)=$ time at nth step, $t_{0}=$ time at start. $t_{4} t_{5}$ represents a variation in step time. ST, step time.

resolved by cross-examination until consensus was reached.

\section{Individual analysis of study bias}

Critical appraisal of literature was conducted throughout the review process, with summaries concluding each section. For assessment of potential sources of bias, and level of evidence for each article based on criteria from the Centre for Evidence Based Medicine (CEBM) $(18,19)$, see the available online: https://cdn.amegroups.cn/static/ public/mhealth-21-7-1.xlsx.

\section{Data extraction}

The following information was collected from each article which met eligibility criteria by 2 independent reviewers ( $\mathrm{CB}$ and $\mathrm{DH})$, and documented available at available online: https://cdn.amegroups.cn/static/public/mhealth-21-7-1.xlsx : 


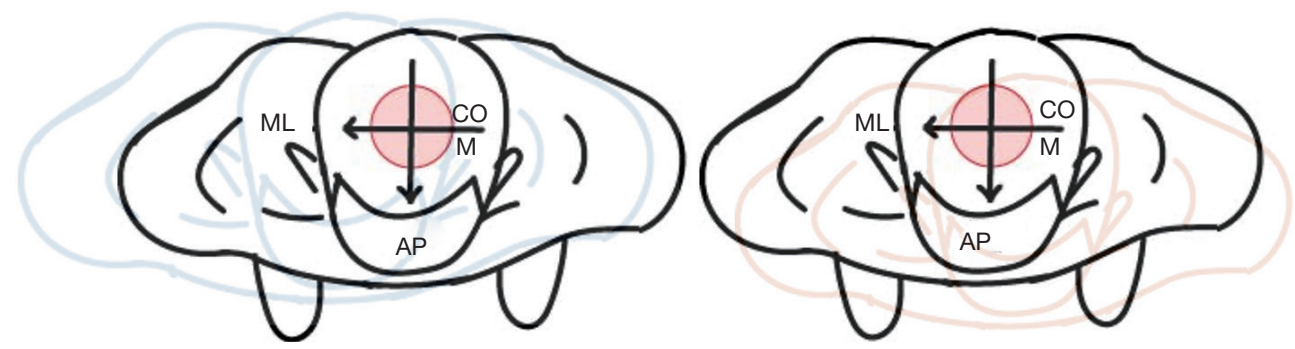

Figure 3 Static balance testing. Blue represents lateral movement of COM relative to the feet, orange represents anterior movement of the COM relative to the feet. ML, medio-lateral; COM, Centre of motion; AP, antero-posterior.

$$
\begin{aligned}
& \text { Gait Velocity }=\frac{\text { Path length }(\text { meters })}{\text { Time taken }(\sec \text { onds })} \\
& \text { Cadence }=\frac{\text { Number of steps }}{\text { Time taken }(\min \text { utes })} \\
& \text { Stride time }=\frac{1}{\text { Cadence }} \\
& \text { Turning steps }=\left|S_{f}{ }_{f}, S_{t 1} S_{t 2} \ldots S_{t n}, S^{\prime \prime}\right| \\
& \text { Turning time }=\text { Time for }\left(S^{\prime}{ }_{f}, S_{t 1} S_{t 2} \ldots S_{t n}, S^{\prime \prime}{ }_{i}\right)
\end{aligned}
$$

Figure 4 Calculations of key gait and balance variables. S'f=final step of first bout, Stn=nth step of turn, S"i=Initial step of second bout.

(I) Number of subjects.

(II) Type, number, and placement of IMU device on subjects.

(III) Gait features that were measured by the IMU device (s) used in the study.

(IV) Length and setting of WB used to measure gait features.

(V) Gait features used in the falls-risk algorithm (if any) produced by the study.

(VI) Tool used to classify subjects into high and low falls-risk group

(VII) Study period over which patients were assessed for falls.

(VIII) Participant demographics, including the presence of any neurological disease affecting gait (NGA).

(IX) General data from the article such as year of publication, journal, objectives, level of evidence, risk of bias and funding sources.

\section{Data synthesis}

Following data collection, studies were sorted into 3 groups pertaining to the barrier to clinical IMU implementation that they addressed, those being device accuracy, device sensitivity, and algorithm predictive accuracy. Qualitative analysis was performed to provide an appreciation of the results of existing literature and discuss whether any barriers remain to implementation.

\section{Results}

\section{Characteristics of included studies}

Since 2002 (20), researchers have investigated the use of single-point or multi-point IMUs to measure gait metrics (Table 2). In total, 46 articles met the eligibility criteria for inclusion in this study (Figure 5). While most used singlepoint IMUs, they examined fewer variables, had more disagreement with gold-standard measurements, and only 5 studied NGAs. Single-point wearables are much easier to use in a clinical setting or home, but they cannot compare data from numerous sensors to reduce false-positive errors in data capture, which may be enhanced in NGAs where movement is less smooth. Inaccuracies also mean that algorithms interpreting the data must provide leeway when identifying steps to avoid false-negative errors in data processing. 17 papers compared IMUs to gold-standard gait-analysis technologies and one (21) assessed test-retest reliability to validate wearables in clinical assessment of relevant gait parameters. 25 papers investigated the ability of IMUs to detect expected gait changes between groups of fallers and age-matched non-fallers. 33 studies used IMUs from $50-100 \mathrm{~Hz}$ to distinguish populations of high and low 
Table 2 Characteristics of studies validating the use of IMU in falls

\begin{tabular}{|c|c|c|c|c|c|c|}
\hline Type of study & Total & $\begin{array}{l}\text { Single } \\
\text { point }\end{array}$ & $\begin{array}{l}\text { Multi } \\
\text {-point }\end{array}$ & Gait metrics studied [n] & Control variables [n] & $\begin{array}{l}\text { Medan CEBM level of evidence } \\
\text { and risk of bias [Range }=a, b]\end{array}$ \\
\hline $\begin{array}{l}\text { Validation against } \\
\text { gold standard }\end{array}$ & 18 & 9 & 9 & $\begin{array}{l}\text { SL [8], GV [7], cadence [4], SC [4], } \\
\text { Turning kinematics [3], dynamic sway } \\
\text { [2], static sway [2], STV [1], SLV [1] }\end{array}$ & $\begin{array}{l}\text { 3D motion capture [8], } \\
\text { gait mats/force plates } \\
\text { [6], videography [5] }\end{array}$ & $\begin{array}{l}\text { 1b }[1 \mathrm{~b}, 4] . \\
\text { Low-moderate } \\
\text { [low, moderate-high] }\end{array}$ \\
\hline Prediction of falls & 12 & 10 & 2 & $\begin{array}{l}\text { SL [3], GV [6], cadence [9], SC [4], } \\
\text { turning kinematics [2], dynamic sway [6], } \\
\text { static sway [1], STV [5], SLV [4] }\end{array}$ & $\mathrm{N} / \mathrm{A}$ & $\begin{array}{l}2 \mathrm{~b}[1 \mathrm{~b}, 4] \\
\text { Low [low, moderate] }\end{array}$ \\
\hline $\begin{array}{l}\text { Classification of } \\
\text { faller/non-faller }\end{array}$ & 13 & 11 & 2 & $\begin{array}{l}\text { SL [2], GV [4], cadence [4], turning } \\
\text { kinematics [1], dynamic sway [7], static } \\
\text { sway [3], STV [1], SLV [1], }\end{array}$ & $\mathrm{N} / \mathrm{A}$ & $\begin{array}{l}4[4,5] \\
\text { Moderate } \\
\text { [low, moderate-high] }\end{array}$ \\
\hline $\begin{array}{l}\text { Classification of } \\
\text { high/low falls risk }\end{array}$ & 6 & 6 & 0 & $\begin{array}{l}\text { GV [1], cadence [5], SC [3], turning } \\
\text { kinematics [1], dynamic sway [5], static } \\
\text { sway [1], STV [2] }\end{array}$ & $\begin{array}{l}\text { TUG [3], PPA [1], BBS } \\
\text { [3], STRATIFY [1], } \\
\text { Tinetti [1], DGI [1] }\end{array}$ & $\begin{array}{l}4[4] \\
\text { Moderate } \\
\text { [low, moderate-high] }\end{array}$ \\
\hline
\end{tabular}

CEBM, Centre for Evidence Based Medicine; GV, gait velocity; SC, daily step count; ST, stride time; SL, stride length; GSV, gait speed variability; STV, stride time variability; SLV, stride length variability; STRATIFY, Ontario Modified Stratify; Tinetti, Tinetti Balance Assessment Tool; DGI, Dynamic Gait Index.

PRISMA 2009 Flow Diagram

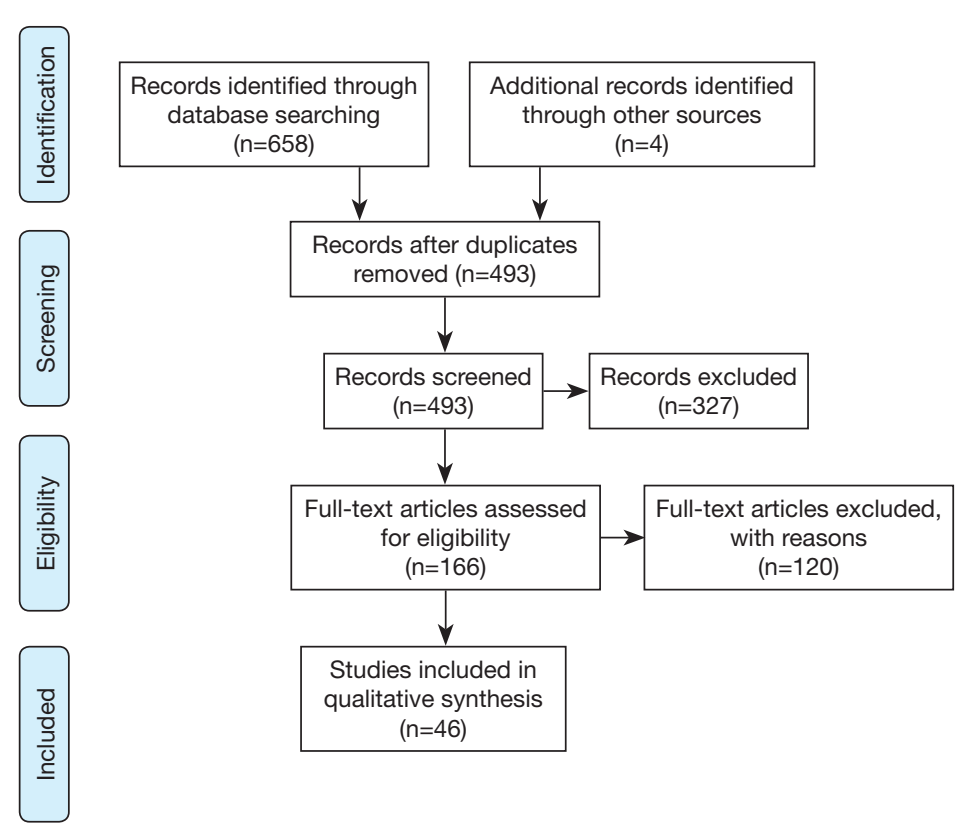

Figure 5 PRISMA flow diagram of the article selection process showing yields at each stage of the process. 
falls-risk determined by fall history (22), test battery score or prospective falls. The 46 studies included in the analysis typically presented with a low-to-moderate risk of bias (available online: https://cdn.amegroups.cn/static/public/ mhealth-21-7-1.xlsx)

\section{Discussion}

\section{Accuracy and reliability in measuring spatiotemporal gait metrics}

Studies using multi-point sensors on the limbs and trunk verified that whether accelerometers were used alone $(23,24)$ or with gyroscopes and magnetometers $(12,25,26)$, they could capture gait with comparable accuracy compared to a gold-standard. GV was accurately detected by multi-point IMUs sampling at $32 \mathrm{~Hz}$ (24) to $500 \mathrm{~Hz}$ (27), SLV and STV at $130 \mathrm{~Hz}(23)$, dynamic balance in ML and VT directions at $130 \mathrm{~Hz}(26)$ and $\mathrm{SC}$ as low as $20 \mathrm{~Hz}$ (12). However, one study (27) found a significant discrepancy between IMU data at $500 \mathrm{~Hz}$ and force-sensitive treadmill data for cadence and SL, while other studies found IMUs $100-200 \mathrm{~Hz}$ to be accurate for cadence (26) and SL $(20,23,24)$. Given otherwise similar methodology and low risk of bias, one possible reason for this discrepancy may be location of sensor placement, which was on the feet in the $500 \mathrm{~Hz}$ trials. This may have caused the IMU to detect noise from smaller movements of the foot during a gait cycle, compared to an IMU placed on the trunk which detects overall motion.

Studies using single-point wearables from $31.26-255 \mathrm{~Hz}$ $(21,28)$ have more disagreement between gold-standard and sensor data. For example, when compared to the Centre of Pressure sway (COP) from a force plate during static posturography, IMU data (sampled at $52 \mathrm{~Hz}$ ) captured from the COM had poor correlation (29), while data capture at $200 \mathrm{~Hz}$ from the sternum highly correlated (30). It is unlikely the difference sampling rates account for this discrepancy (given both are above the Nyquist sampling thresholds of around $30 \mathrm{~Hz}$ for detecting human motion) (31). Perhaps placing the IMU above the COM magnifies small movements in the $\mathrm{AP}$ and $\mathrm{ML}$ directions, thus being able to distinguish sway from noise. This translates into the sternum being a key location for single-point fixation wearables for gait and falls assessment. When single-point sensors were used to measure SL $(8,28,32)$, cadence $(8,28), \mathrm{GV}(8)$, SC $(28,33)$, STV (8), dynamic sway (34) and turning (35), they demonstrated good-to-excellent agreement with goldstandard methods of gait analysis. The findings of these studies thus suggest the gait metrics measured by wearables have sufficient accuracy and reliability to be used in clinical setting, but the consistency of this agreement from condition-to-condition is yet to be assessed.

\section{Sensitivity in detecting differences between fallers and non-fallers}

Regarding the ability to differentiate between groups of age- and disease-matched fallers and non-fallers, studies tended to show that wearable devices were able to detect subtle differences in relevant biomechanical gait metrics between these groups. Overall, IMUs were sensitive enough to detect differences in GV $(25,36-42)$, SL $(25,37,39,40)$, turning kinematics $(25,41,43)$, GSV (39), SLV (37), STV $(36,44)$, cadence $(25,36,39-41,45)$ and postural sway in single $(39,46,47)$ or multiple axes $(25,38,40-42,48-50)$ between fallers and non-fallers. Only one retrospective study did not identify dynamic imbalance during treadmill walking as significantly different between fallers and non-fallers (51). However, it should be noted that past fallers tend to consciously widen their stance for lateral stability, and this effect is enhanced on a treadmill (52). Moreover, this study had a moderate-high risk of bias according to CEBM criteria. Interestingly, most studies assessing SC using single-point IMUs at $50-100 \mathrm{~Hz}$, did not find that fallers had a significantly lower SC than nonfallers $(37,39,44,49,50,53)$, while one did (40). This may be because waist, thigh and wrist-worn high-frequency single-point sensors underestimate SC by $2-10 \%$ (54) and SC seems to only be altered in fallers with NGAs, which only one study (50) assessed. IMU devices accurately measure gait factors related to falls-risk and appear able to differentiate between groups of fallers and non-fallers using these factors. However, for clinical implementation this sensitivity in detecting differences in gait metrics needs to be confirmed in continuous real-world data collection in home and community environments.

\section{Classification accuracy of falls-prediction tools}

Regarding the accuracy of falls-prediction algorithms developed based on biomechanical data collected by IMUs, studies were considered successful if biomechanical data was used by an algorithm to accurately sort patients into 
high- and low-falls-risk groups. In successful studies, these algorithms were developed post-hoc by neural network, machine learning or decision trees, trialling sets of 5-30 gait parameters to determine the most successful combination. Limiting the number of gait parameters trialled at once prevents 'over-training', which increases internal classification accuracy but reduces generalisability (55). Nonetheless, these post-hoc selected 'inputs' into the algorithms increases risk of a 'contextualised' model and its accuracy, reliability and validity to the generalised population of fallers is unknown without validation in external data-sets.

Additionally, there is limited evidence verifying the role of static balance in falls, several papers utilising biomechanical data algorithms to classify fallers performed static posturography. 5 papers utilised static postural stability in their algorithm yielding faller classification accuracies of $71.2-85 \%(25,56,57)$ and falls-risk classification accuracies of $90.5 \%$ (36) and $97 \%$ (58) to Tinetti and STRATIFY scores respectively. While their results seem impressive, it should be noted that multiple measures like SD and CV were used for sway, and the algorithm tested combinations of them until it yielded the highest classification accuracy. By inputting the same variable multiple times, false-positive results are more likely, and this can only be accounted for by testing the algorithm against novel data to test true classification accuracy, known as 'cross-validation' (24,59). The majority of these papers had a moderate risk of bias according to CEBM criteria (36,56-58).

That said, when papers did not include static posturography, classification of fallers was still accomplished. A study by Iluz et al., (38) using a $100 \mathrm{~Hz}$ IMU at the COM identified lower GV, and higher sway in AP and VT directions during daily-life sit-to-walk transitions in fallers compared to age-matched adults. Combining this data into a faller identification algorithm yielded a classification accuracy of $88 \%$ however, using data captured during labbased gait yielded $71 \%$ accuracy. This may imply that algorithms should be specific to the gait-capture setting. Iluz's paper is one of many to combine dynamic sway with metrics including GV $(25,47,60)$, SL $(25,60)$, turning kinematics $(25,59)$, cadence $(25,59-62)$, STV $(60,63)$, SLV (60) and GSV (60) in algorithms with moderategood classification accuracies of $68-85 \%$, consistently outperforming existing test batteries $(36,38,43,47,56,57)$. One study (64) used cadence, SL and GV measured by a triaxial accelerometer at $100 \mathrm{~Hz}$ at the COM in an algorithm which classified 6-month prospective fallers with an Odds-Ratio of
3.89 if the cut-off value was exceeded. An algorithm using SLV, cadence and turning kinematics from $102.4 \mathrm{~Hz}$ sensors at each shank had a predictive accuracy of 2-year prospective falls of $83.02 \%$ and another algorithm using SL, STV and turning kinematics had an accuracy of $72.97 \%$ (43).

\section{Feasibility and limitations}

No studies thus far have attempted falls-risk classification using IMU data from NGA populations. As such, limitations still exist when it comes to the assessment of falls-risk in neurological populations which make up a large portion of hospital inpatients and clinical visitors. Falls-classification tools in elderly populations used singleor multi-point IMUs with various sensor configurations, walking settings and gait-metrics. Although, IMU devices are able to assign falls risk using algorithms developed with gait metrics collected from WBs for non-neurological populations, these are yet to be extensively validated in large external populations. Current falls-prediction tools therefore require validation in external data-sets before clinical implementation Moreover current classification accuracy is limited by comparison against self-report of retrospective falls (over a set period) by patients which is not an accurate nor reliable gold-standard of objective measurement. Additionally, falls-prediction tools constructed from 'walking bouts' may not reflect falls-risk during 'free-living' gait and thus future studies employing gait metrics from continuous monitoring of walking in the community and in the home are warranted.

Despite these limitations, IMUs placed at the COM, chest or limbs, sampling at $50-250 \mathrm{~Hz}$ have been validated against gold-standard methods in capturing various gait metrics. They also have the potential to distinguish fallers and non-fallers based on gait deterioration patterns and use this to accurately assign falls-risk. Single-point wearables are more viable in clinical settings but have not been thoroughly tested in NGA populations nor validated for SLV, static sway or GSV. Moreover, the studies have a lot of heterogeneity in methodology, with no clear standard. Finally, our review included insufficient studies of NGA populations and more data is required to justify using single-point IMUs for gait analysis and falls prediction in NGAs, primarily those of spinal origin.

\section{Conclusions}

Currently, the literature is divided on metrics like GV 
and SL as predictors of falls, and advents such as turning kinetics are in the immature stage of research. In addition, the bulk of studies examine community-dwelling elderly, with few investigating NGAs and none investigating spinal NGAs. Moreover, while the use of sensor technology in falls-risk analysis has been investigated, the studies examine very few gait metrics at once, and comparing studies reveals a wide variety of sensor types in use. Future avenues of research thus include investigations of multiple gait metrics simultaneously especially in NGA and spinal NGA populations.

\section{Acknowledgments}

Special thanks to Dr Greg Smith for helping to develop the content and structure of the manuscript. Doctor Matthew Brodie for providing details of past publications and proofreading the section on wearable technology. Luke Sy for algorithm and application development for the methodology. Jessie Ding for creating the artwork used in this manuscript.

Funding: This work did not receive funding from any organisations. The work was done as part of a research project for the University of New South Wales, Department of Medicine (NSW, Australia), in collaboration with NeuroSpineClinic (Suite 7 Level 7, Prince of Wales Private Hospital, NSW, Australia) and the NSURG Research Group (Level 7, Prince of Wales Private Hospital, NSW, Australia).

\section{Footnotes}

Reporting Checklist: The authors have completed the Narrative Review reporting checklist. Available at https:// dx.doi.org/10.21037/mhealth-21-7

Conflicts of Interest: All authors have completed the ICMJE uniform disclosure form (available at https://dx.doi. org/10.21037/mhealth-21-7). The authors have no conflicts of interest to declare

Ethical Statement: The authors are accountable for all aspects of the work in ensuring that questions related to the accuracy or integrity of any part of the work are appropriately investigated and resolved.

Open Access Statement: This is an Open Access article distributed in accordance with the Creative Commons
Attribution-NonCommercial-NoDerivs 4.0 International License (CC BY-NC-ND 4.0), which permits the noncommercial replication and distribution of the article with the strict proviso that no changes or edits are made and the original work is properly cited (including links to both the formal publication through the relevant DOI and the license). See: https://creativecommons.org/licenses/by-nc-nd/4.0/.

\section{References}

1. Australian Institute of Health and Welfare. Injury. Canberra, Australia: 2019.

2. World Health Organisation. Falls. World Health Organisation, Geneva, Switzerland. 2018. Available online: https://www.who.int/news-room/fact-sheets/detail/falls

3. McCrum C, Gerards MHG, Karamanidis K, et al. A systematic review of gait perturbation paradigms for improving reactive stepping responses and falls risk among healthy older adults. Eur Rev Aging Phys Act 2017;14:3.

4. Ronthal M. Neurologic gait disorders of elderly people. UpToDate. 2018. Available online: https://www.uptodate. com/contents/neurologic-gait-disorders-of-elderly-people

5. Stolze H, Klebe S, Zechlin C, et al. Falls in frequent neurological diseases--prevalence, risk factors and aetiology. J Neurol 2004;251:79-84.

6. Gor-García-Fogeda MD, Cano de la Cuerda R, Carratalá Tejada M, et al. Observational Gait Assessments in People With Neurological Disorders: A Systematic Review. Arch Phys Med Rehabil 2016;97:131-40.

7. Toro B, Nester C, Farren P. A review of observational gait assessment in clinical practice. Physiother Theory Pract 2003;19:137-49.

8. Brodie MA, Coppens MJ, Lord SR, et al. Wearable pendant device monitoring using new wavelet-based methods shows daily life and laboratory gaits are different. Med Biol Eng Comput 2016;54:663-74.

9. Majumder S, Mondal T, Deen MJ. Wearable Sensors for Remote Health Monitoring. Sensors (Basel) 2017;17:130.

10. Vienne A, Barrois RP, Buffat S, et al. Inertial Sensors to Assess Gait Quality in Patients with Neurological Disorders: A Systematic Review of Technical and Analytical Challenges. Front Psychol 2017;8:817.

11. Patonis P, Patias P, Tziavos IN, et al. A Fusion Method for Combining Low-Cost IMU/Magnetometer Outputs for Use in Applications on Mobile Devices. Sensors (Basel) 2018;18:2616.

12. Anwary AR, Yu H, Vassallo M. An Automatic Gait Feature 
Extraction Method for Identifying Gait Asymmetry Using Wearable Sensors. Sensors (Basel) 2018;18:676.

13. Creaby MW, Cole MH. Gait characteristics and falls in Parkinson's disease: A systematic review and meta-analysis. Parkinsonism Relat Disord 2018;57:1-8.

14. Fasano A, Canning CG, Hausdorff JM, et al. Falls in Parkinson's disease: A complex and evolving picture. Mov Disord 2017;32:1524-36.

15. Gillain S, Boutaayamou M, Beaudart C, et al. Assessing gait parameters with accelerometer-based methods to identify older adults at risk of falls: a systematic review. Eur Geriatr Med 2018;9:435-48.

16. Mortaza N, Abu Osman NA, Mehdikhani N. Are the spatio-temporal parameters of gait capable of distinguishing a faller from a non-faller elderly? Eur J Phys Rehabil Med 2014;50:677-91.

17. Menant JC, Schoene D, Sarofim M, et al. Single and dual task tests of gait speed are equivalent in the prediction of falls in older people: a systematic review and meta-analysis. Ageing Res Rev 2014;16:83-104.

18. Phillips B, Ball C, Sackett D, et al. Oxford Centre for Evidence-based Medicine - Levels of Evidence (March 2009). Centre for Evidence Based Medicine, Oxford, UK. 2009. Available online: https://www.cebm.net/2009/06/ oxford-centre-evidence-based-medicine-levels-evidencemarch-2009/

19. Centre for Evidence Based Medicine. Critical Appraisal tools. University of Oxford, Oxford, UK. 2014.

Available online: https://www.cebm.net/2014/06/ critical-appraisal/

20. Aminian K, Najafi B, Büla C, et al. Spatio-temporal parameters of gait measured by an ambulatory system using miniature gyroscopes. J Biomech 2002;35:689-99.

21. Saunders NW, Koutakis P, Kloos AD, et al. Reliability and validity of a wireless accelerometer for the assessment of postural sway. J Appl Biomech 2015;31:159-63.

22. Skelton D, Todd C. What are the main risk factors for falls amongst older people and what are the most effective interventions to prevent these falls? Copenhagen: World Health Organisation, Network HE;2004.

23. Rebula JR, Ojeda LV, Adamczyk PG, et al. Measurement of foot placement and its variability with inertial sensors. Gait Posture 2013;38:974-80.

24. Aich S, Pradhan PM, Park J, et al. A Validation Study of Freezing of Gait (FoG) Detection and MachineLearning-Based FoG Prediction Using Estimated Gait Characteristics with a Wearable Accelerometer. Sensors (Basel) 2018;18:3287.
25. Qiu H, Rehman RZU, Yu X, et al. Application of Wearable Inertial Sensors and A New Test Battery for Distinguishing Retrospective Fallers from Non-fallers among Community-dwelling Older People. Sci Rep 2018;8:16349.

26. Brodie MA, Beijer TR, Canning CG, et al. Head and pelvis stride-to-stride oscillations in gait: validation and interpretation of measurements from wearable accelerometers. Physiol Meas 2015;36:857-72.

27. Donath L, Faude O, Lichtenstein E, et al. Mobile inertial sensor based gait analysis: Validity and reliability of spatiotemporal gait characteristics in healthy seniors. Gait Posture 2016;49:371-4.

28. O'Brien MK, Hidalgo-Araya MD, Mummidisetty CK, et al. Augmenting Clinical Outcome Measures of Gait and Balance with a Single Inertial Sensor in Age-Ranged Healthy Adults. Sensors (Basel) 2019;19:4537.

29. Jebelli H, Ahn CR, Stentz TL. Fall risk analysis of construction workers using inertial measurement units: Validating the usefulness of the postural stability metrics in construction. Saf Sci 2016;84:161-70.

30. Hsieh KL, Roach KL, Wajda DA, et al. Smartphone technology can measure postural stability and discriminate fall risk in older adults. Gait Posture 2019;67:160-5.

31. Cheng Q, Juen J, Li Y, et al. editors. GaitTrack: Health monitoring of body motion from spatio-temporal parameters of simple smart phones. Proceedings of the International Conference on Bioinformatics, Computational Biology and Biomedical Informatics, 2013.

32. Kitagawa N, Ogihara N. Estimation of foot trajectory during human walking by a wearable inertial measurement unit mounted to the foot. Gait Posture 2016;45:110-4.

33. Najafi B, Armstrong DG, Mohler J. Novel wearable technology for assessing spontaneous daily physical activity and risk of falling in older adults with diabetes. J Diabetes Sci Technol 2013;7:1147-60.

34. Brodie MA, Psarakis M, Hoang P. Gyroscopic corrections improve wearable sensor data prior to measuring dynamic sway in the gait of people with Multiple Sclerosis. Comput Methods Biomech Biomed Engin 2016;19:1339-46.

35. El-Gohary M, Pearson S, McNames J, et al. Continuous monitoring of turning in patients with movement disability. Sensors (Basel) 2013;14:356-69.

36. Gietzelt M, Feldwieser F, Gövercin M, et al. A prospective field study for sensor-based identification of fall risk in older people with dementia. Inform Health Soc Care 
2014;39:249-61.

37. Del Din S, Galna B, Godfrey A, et al. Analysis of FreeLiving Gait in Older Adults With and Without Parkinson's Disease and With and Without a History of Falls: Identifying Generic and Disease-Specific Characteristics. J Gerontol A Biol Sci Med Sci 2019;74:500-6.

38. Iluz T, Weiss A, Gazit E, et al. Can a Body-Fixed Sensor Reduce Heisenberg's Uncertainty When It Comes to the Evaluation of Mobility? Effects of Aging and Fall Risk on Transitions in Daily Living. J Gerontol A Biol Sci Med Sci 2016;71:1459-65.

39. van Schooten KS, Pijnappels M, Rispens SM, et al. Daily-Life Gait Quality as Predictor of Falls in Older People: A 1-Year Prospective Cohort Study. PLoS One 2016;11:e0158623.

40. van Schooten KS, Pijnappels M, Rispens SM, et al. Ambulatory fall-risk assessment: amount and quality of daily-life gait predict falls in older adults. J Gerontol A Biol Sci Med Sci 2015;70:608-15.

41. Zakaria NA, Kuwae Y, Tamura T, et al. Quantitative analysis of fall risk using TUG test. Comput Methods Biomech Biomed Engin 2015;18:426-37.

42. Brodie MA, Menz HB, Smith ST, et al. Good lateral harmonic stability combined with adequate gait speed is required for low fall risk in older people. Gerontology 2015;61:69-78.

43. Greene BR, Doheny EP, Walsh C, et al. Evaluation of falls risk in community-dwelling older adults using body-worn sensors. Gerontology 2012;58:472-80.

44. Brodie MA, Coppens MJ, Ejupi A, et al. Comparison between clinical gait and daily-life gait assessments of fall risk in older people. Geriatr Gerontol Int 2017;17:2274-82.

45. Schwesig R, Fischer D, Lauenroth A, et al. Can falls be predicted with gait analytical and posturographic measurement systems? A prospective follow-up study in a nursing home population. Clin Rehabil 2013;27:183-90.

46. Mahoney JR, Oh-Park M, Ayers E, et al. Quantitative trunk sway and prediction of incident falls in older adults. Gait Posture 2017;58:183-7.

47. Isho T, Tashiro H, Usuda S. Accelerometry-based gait characteristics evaluated using a smartphone and their association with fall risk in people with chronic stroke. J Stroke Cerebrovasc Dis 2015;24:1305-11.

48. Bischoff-Ferrari HA, Conzelmann M, Stähelin HB, et al. Is fall prevention by vitamin $\mathrm{D}$ mediated by a change in postural or dynamic balance? Osteoporos Int 2006;17:656-63.
49. Weiss A, Brozgol M, Dorfman M, et al. Does the evaluation of gait quality during daily life provide insight into fall risk? A novel approach using 3-day accelerometer recordings. Neurorehabil Neural Repair 2013;27:742-52.

50. Weiss A, Herman T, Giladi N, et al. Objective assessment of fall risk in Parkinson's disease using a body-fixed sensor worn for 3 days. PLoS One 2014;9:e96675.

51. Riva F, Toebes MJ, Pijnappels $M$, et al. Estimating fall risk with inertial sensors using gait stability measures that do not require step detection. Gait Posture 2013;38:170-4.

52. Tesio L, Rota V. The Motion of Body Center of Mass During Walking: A Review Oriented to Clinical Applications. Front Neurol 2019;10:999.

53. Brodie MA, Okubo Y, Annegarn J, et al. Disentangling the health benefits of walking from increased exposure to falls in older people using remote gait monitoring and multi-dimensional analysis. Physiol Meas 2017;38:45-62.

54. Bassett DR Jr, Toth LP, LaMunion SR, et al. Step Counting: A Review of Measurement Considerations and Health-Related Applications. Sports Med 2017;47:1303-15.

55. Pham HNA, Triantaphyllou E. The Impact of Overfitting and Overgeneralization on the Classification Accuracy in Data Mining. In: Maimon O, Rokach L. editors. Soft Computing for Knowledge Discovery and Data Mining. Boston, MA: Springer US; 2008:391-431.

56. Greene BR, McGrath D, Walsh L, et al. Quantitative falls risk estimation through multi-sensor assessment of standing balance. Physiol Meas 2012;33:2049-63.

57. Greene BR, Doheny EP, Kenny RA, et al. Classification of frailty and falls history using a combination of sensor-based mobility assessments. Physiol Meas 2014;35:2053-66.

58. Giansanti D, Macellari V, Maccioni G. New neural network classifier of fall-risk based on the Mahalanobis distance and kinematic parameters assessed by a wearable device. Physiol Meas 2008;29:N11-9.

59. Drover D, Howcroft J, Kofman J, et al. Faller Classification in Older Adults Using Wearable Sensors Based on Turn and Straight-Walking Accelerometer-Based Features. Sensors (Basel) 2017;17:1321.

60. Ihlen EAF, van Schooten KS, Bruijn SM, et al. Improved Prediction of Falls in Community-Dwelling Older Adults Through Phase-Dependent Entropy of Daily-Life Walking. Front Aging Neurosci 2018;10:44.

61. Howcroft J, Lemaire ED, Kofman J. Wearable-Sensor- 
Based Classification Models of Faller Status in Older Adults. PLoS One 2016;11:e0153240.

62. Rivolta MW, Aktaruzzaman M, Rizzo G, et al. Evaluation of the Tinetti score and fall risk assessment via accelerometry-based movement analysis. Artif Intell Med 2019;95:38-47.

63. Gietzelt M, Nemitz G, Wolf KH, et al. A clinical study to doi: $10.21037 /$ mhealth-21-7

Cite this article as: Betteridge CMW, Natarajan P, Fonseka RD, Ho D, Mobbs R, Choy WJ. Objective falls-risk prediction using wearable technologies amongst patients with and without neurogenic gait alterations: a narrative review of clinical feasibility. mHealth 2021;7:61. assess fall risk using a single waist accelerometer. Inform Health Soc Care 2009;34:181-8.

64. Mignardot JB, Deschamps T, Barrey E, et al. Gait disturbances as specific predictive markers of the first fall onset in elderly people: a two-year prospective observational study. Front Aging Neurosci 2014;6:22. 


\section{Appendix 1 Research question}

A search of the literature was conducted to address the research question "What is the current use of wearable technologies for falls-risk assessment" as shown in the PICO format below. A search strategy was created to identify relevant studies based on the research question below (set out in the PICO format):

\begin{tabular}{ll}
\hline Population & Falls-risk individuals in both neurological and non-neurological populations \\
Intervention & Wearable technology based gait analysis tools for fall-risk assessment \\
Control (Reference Clinical Tool) & Other clinical fall-risk assessment tools based on gait analysis e.g. laboratory-based 3D motion \\
Outcome & Successful/accurate falls-risk prediction \\
\hline
\end{tabular}

A search of Medline, PubMed, Scopus and Embase databases was performed in February 2020. The complete search strategy can be obtained from the attached PROSPERO registration (ID: CRD42020195861)

\section{Included Studies:}

Medline, Embase, Pubmed and Scopus were systematically searched from their date of inception to February, 2020. A manual search for other relevant articles was also conducted by examining the references and citations of key papers. Database and bibliographic search identified 662 relevant studies. After removal of duplicates, 493 studies remained. 327 references were excluded on title and abstract screen and 120 references were excluded by full-text analysis, leaving a final 46 studies to be included in qualitative synthesis (see Figure 5 for PRISMA flow chart). Reasons for exclusion during full text review include: not involving falls-risk prediction tool/model (73), not involving wearable technologies (47).

\section{Appendix 2 Review registration}

Do fallers walk funny? A systematic review of gait metrics that predict falls in high-risk populations Callum Betteridge, Daniel Ho

To enable PROSPERO to focus on COVID-19 registrations during the 2020 pandemic, this registration record was automatically published exactly as submitted. The PROSPERO team has not checked eligibility.

\section{Citation}

Callum Betteridge, Daniel Ho. Do fallers walk funny? A systematic review of gait metrics that predict falls in high-risk populations. PROSPERO 2020 CRD42020195861 Available from: https://www.crd.york.ac.uk/prospero/display_record. php?ID=CRD42020195861

\section{Review question}

P: In adult patients (normal or neurogenic gait alterations) at risk of falls/with a history of falls

I: Which aspects of gait or posture change

C: Compared to adult patients without falls risk/history of falls

O: And are predictive of falls risk

\section{Searches}

MEDLINE, PubMed, EMBASE, Scopus, Search date 28/02/2020

\section{Search strategy}

https://www.crd.york.ac.uk/PROSPEROFILES/195861_STRATEGY_20200628.pdf

\section{Types of study to be included}

Cohort or case control

\section{Condition or domain being studied}

Risk of falls in adults with and without neurological disease

\section{Participants/population}

Adult fallers versus non-fallers, with or without neurological disease

\section{Intervention(s), exposure(s)}

Changed gait parameters 


\section{Comparator(s)/control}

Gait parameters in non-fall patients

\section{Main outcome(s)}

Main outcome is either history of falls or prospective falls events

\section{* Measures of effect}

Relative risks and odds ratios

\section{Additional outcome(s)}

None

\section{* Measures of effect}

None

\section{Data extraction (selection and coding)}

Papers are cohort, with 6 or greater month follow up, or case-control studies comparing groups of age and/or diseasematched fallers and non-fallers with validated methods of gait analysis and be published after January 1st, 2015.

Data collected from articles post-screening documenting which gait variables are measured, and their effects on risk of falls. Additionally, how gait metrics and falls risk were assessed, patient numbers and broad demographics (esp whether or not there was a neurological alteration to gait)

\section{Risk of bias (quality) assessment}

Risk of bias and level of evidence for each article is assessed using the Oxford University Centre for Evidence Based Medicine (CEBM) criteria

\section{Strategy for data synthesis}

If consistent measures of falls-risk and gait metrics are used, data will be synthesised assuming that there are more than 3 studies for that gait metric.

Method will be using forest plots if this is available.

\section{Analysis of subgroups or subsets}

'Subgroups' will be defined by each gait metric, for example for gait velocity, any paper studying it will be examined and the results combined if gait velocity is measured consistently, if falls-risk is measured consistently, and if there are 3 or more applicable studies.

\section{Contact details for further information}

Callum Betteridge

cbetteridgeresearch@gmail.com

\section{Organisational affiliation of the review}

University of New South Wales

https://www.unsw.edu.au/

\section{Review team members and their organisational affiliations}

Mr Callum Betteridge. University of New South Wales

Mr Daniel Ho. University of New South Wales

\section{Collaborators}

Professor Ralph Mobbs. University of New South Wales Mr Wen Jie Choy. University of New South Wales

\section{Type and method of review}

Diagnostic, Prognostic, Systematic review 


\section{Anticipated or actual start date}

01 March 2020

\section{Anticipated completion date}

30 July 2020

\section{Funding sources/sponsors}

No funding was obtained for this review, it was performed independently

\section{\#Conflicts of interest}

\section{Language}

English

\section{Country}

Australia

\section{Stage of review}

Review Ongoing

\section{Subject index terms status}

Subject indexing assigned by CRD

\section{Subject index terms}

$\mathrm{MeSH}$ headings have not been applied to this record

\section{Date of registration in PROSPERO}

30 July 2020

\section{Date of first submission}

28 June 2020

\section{Stage of review at time of this submission}

\begin{tabular}{lcc}
\hline Stage & Started & Completed \\
\hline Preliminary searches & Yes & Yes \\
Piloting of the study selection process & Yes & Yes \\
Formal screening of search results against eligibility criteria & Yes & Yes \\
Data extraction & No & No \\
Risk of bias (quality) assessment & No & No \\
Data analysis & No & No
\end{tabular}

The record owner confirms that the information they have supplied for this submission is accurate and complete and they understand that deliberate provision of inaccurate information or omission of data may be construed as scientific misconduct.The record owner confirms that they will update the status of the review when it is completed and will add publication details in due course. 